\title{
ON FIXED POINT THEOREMS OF CONTRACTIVE TYPE
}

\author{
MAU-HSIANG SHIH AND CHEH-CHIH YEH
}

\begin{abstract}
Let $G$ be a continuous map of a nonempty compact metric space $(X, d)$ into itself, such that for some positive integer $m$, the iterated map $G^{m}$ satisfying

$$
\begin{aligned}
& d\left(G^{m}(x), G^{m}(y)\right) \\
& \quad<\max \left\{d(x, y), d\left(x, G^{m}(x)\right), d\left(y, G^{m}(y)\right), d\left(x, G^{m}(y)\right), d\left(y, G^{m}(x)\right)\right\}
\end{aligned}
$$

for all $x, y \in X$ with $x \neq y$. It is shown that (i) $G$ has a unique fixed point $x^{*} \in X$; (ii) the sequence of iterates $\left\{G^{k}(x)\right\}$ converges to $x^{*}$ for any $x \in X$; (iii) given $\lambda, 0<\lambda<1$, there exists a metric $d_{\lambda}$, topologically equivalent to $d$, such that $d_{\lambda}(G(x), G(y)) \leq \lambda d_{\lambda}(x, y)$ for all $x, y \in X$.
\end{abstract}

1. Among the various extensions of the contraction mapping principle, an important and simple one is the Kolmogoroff and Fomin theorem (KFT) [7, pp. 160-161]. The KFT asserts that if $G$ is a continuous map of a complete metric space $(X, d)$ into itself, such that for some positive integer $m$, the iterated map $G^{m}$ is a contraction (in the sense of Banach), then $G$ has a unique fixed point in $X$. Chu and Diaz [2] then gave a thorough analysis of KFT and pointed out various possible modifications. Note that KFT is particularly useful for Volterra integral equations [7]. In a recent paper Ćirić proved a result [3, Theorem 2] which may be called a generalized KFT. It is the purpose of this note to look at the generalized KFT from another point of view, namely, remetrization theory.

2. We begin with the following theorem, of which parts (a), (b) and (c) were already proved in $[\mathbf{3}]$.

THEOREM 1. Let $G$ be a map of a (nonempty) complete metric space $(X, d)$ into itself, such that for some positive integer $m$, the iterated map $G^{m}$ satisfying

$$
\begin{aligned}
& d\left(G^{m}(x), G^{m}(y)\right) \\
& \quad \leq \alpha \max \left\{d(x, y), d\left(x, G^{m}(x)\right), d\left(y, G^{m}(y)\right), d\left(x, G^{m}(y)\right), d\left(y, G^{m}(x)\right)\right\}
\end{aligned}
$$

for some $\alpha \in(0,1)$ and for all $x, y \in X$. Then

(a) $G$ has a unique fixed point $x^{*} \in X$;

(b) $\lim _{k \rightarrow \infty} G^{k}(x)=x^{*}$ for all $x \in X$;

(c) $d\left(G^{k}(x), x^{*}\right) \leq \alpha^{j} a(x) /(1-\alpha)$ for all $x \in X$,

where $a(x):=\max \left\{d\left(G^{i}(x), G^{i+m}(x)\right): i=0,1, \ldots, m-1\right\}$ and $j:=[k / m]$ is the greatest integer not exceeding $k / m$.

Moreover, assume further that $G$ is continuous, then we have

(d) given $\lambda \in(0,1)$, there exists a metric $d_{\lambda}$, topologically equivalent to $d$, such that $d_{\lambda}(G(x), G(y)) \leq \lambda d_{\lambda}(x, y)$ for all $x, y \in X$.

Received by the editors June 24, 1981 and, in revised form, October 22, 1981.

1980 Mathematics Subject Classification. Primary 54H25.

Key words and phrases. Contraction, fixed point, remetrization. 
PROOF. For proofs of (a), (b) and (c), see [3, Theorem 2]. To prove (d) we shall make use of Meyer's theorem [8].

MEYER'S THEOREM. Let $G$ be a continuous map of a (nonempty) metric space $(X, d)$ into itself such that

(i) $G$ has a unique fixed point $x^{*} \in X$;

(ii) the sequence of iterates $\left\{G^{k}(x)\right\}$ converges to $x^{*}$ for all $x \in X$;

(iii) there exists an open neighborhood $U$ of $x^{*}$ with the property that given any open set $V$ containing $x^{*}$ there exists a positive integer $N$ such that $G^{k}(U) \subset V$ for all $k \geq N$.

Then for any $\lambda \in(0,1)$, there exists a metric $d_{\lambda}$, topologically equivalent to $d$, such that

$$
d_{\lambda}(G(x), G(y)) \leq \lambda d_{\lambda}(x, y) \quad \text { for all } x, y \in X
$$

Proof of TheOREM 1 (CONTINUED). By the conclusion of (a) and (b), and Meyer's theorem, we have to find an open neighborhood $U$ of $x^{*}$ such that $\left\{G^{k}(U)\right\}$ converges to $x^{*}$. Let

$$
U:=\left\{x \in X: d\left(x, x^{*}\right)<1\right\} .
$$

Then by inequality (c), $G^{k}(U) \rightarrow x^{*}$. This completes the proof.

The following theorem solves the case $\alpha=1$ in Theorem 1 .

THEOREM 2. Let $G$ be a continuous map of a nonempty compact metric space $(X, d)$ into itself, such that for some positive integer $m$, the iterated map $G^{m}$ satisfying

$$
\begin{aligned}
d\left(G^{m}(x), G^{m}(y)\right) & \\
& <\max \left\{d(x, y), d\left(x, G^{m}(x)\right), d\left(y, G^{m}(y)\right), d\left(x, G^{m}(y)\right), d\left(y, G^{m}(x)\right)\right\}
\end{aligned}
$$

for all $x, y \in X$ with $x \neq y$. Then

(a) $G$ has a unique fixed point $x^{*} \in X$;

(b) the sequence of iterates $\left\{G^{k}(x)\right\}$ converges to $x^{*}$ for any $x \in X$;

(c) given $\lambda \in(0,1)$, there exists a metric $d_{\lambda}$, topologically equivalent to $d$, such that

$$
d_{\lambda}(G(x), G(y)) \leq \lambda d_{\lambda}(x, y) \quad \text { for all } x, y \in X
$$

Proof. Let $G^{m}:=F$. Note that $F^{k}(X) \supset F^{k+1}(X)$ for $k=1,2, \ldots$ From the compactness of $X$ and the continuity of $F$, it follows that $\left\{F^{k}(X)\right\}$ has the finite intersection property; thus $\bigcap_{k=1}^{\infty} F^{k}(X)$ is a nonempty compact subset of $X$. Let $H:=\bigcap_{k=1}^{\infty} F^{k}(X)$. We shall prove that $F(H)=H$. It is clear that $F(H) \subset H$. To obtain this reverse part, let $x \in H$; then there exists $x_{k} \in F^{k}(X)$ such that $F\left(x_{k}\right)=x, k=1,2, \ldots$ By compactness of $X$ we may (by selecting a subsequence, if necessary) suppose that $x_{k}$ converges to some point $\bar{x} \in X$. Since $\left\{x_{k}, x_{k+1}, \ldots\right\} \subset F^{k}(X)$ and $F^{k}(X)$ is compact, $\bar{x} \in F^{k}(X)$ for $k=1,2, \ldots$ So that $\bar{x} \in H$ and by $F(\bar{x})=x$, we have $H \subset F(H)$. This obtains our desired conclusion. Moreover, $\delta(H)=0$, where $\delta(H)$ denotes the diameter of $H$. Suppose not. Since the product space $H \times H$ is a nonempty compact set, by the continuity of $d$, there exist two distinct points $z_{1}, z_{2} \in H$ such that $\delta(H)=d\left(z_{1}, z_{2}\right)$. From $F(H)=H$, it follows that there are two distinct points $x_{1}, x_{2} \in H$ such that 


$$
\begin{aligned}
& F\left(x_{1}\right)=z_{1} \text { and } F\left(x_{2}\right)=z_{2} . \text { But } \\
& \begin{aligned}
\delta(H) & =d\left(F\left(x_{1}\right), F\left(x_{2}\right)\right) \\
& \leq \max \left\{d\left(x_{1}, x_{2}\right), d\left(x_{1}, F\left(x_{1}\right)\right), d\left(x_{2}, F\left(x_{2}\right)\right), d\left(x_{1}, F\left(x_{2}\right)\right), d\left(x_{2}, F\left(x_{1}\right)\right)\right\}
\end{aligned}
\end{aligned}
$$

a contradiction to (2). So $H$ is a singleton, say, $H=\left\{x^{*}\right\}$. Therefore $F^{k}(x) \rightarrow x^{*}$ for all $x \in X$ and $x^{*}$ is the unique fixed point of $F$. So $G^{m}\left(G\left(x^{*}\right)\right)=G\left(x^{*}\right)$ and the uniqueness of the fixed point of $G^{m}$ leads to $G\left(x^{*}\right)=x^{*}$ and $x^{*}$ is the unique fixed point of $G$. This proves (a). To prove (b) and (c), observe that for $i=0,1, \ldots, m-1$,

$$
\begin{aligned}
\emptyset & \neq \bigcap_{k=1}^{\infty} G^{k m}\left(G^{i}(X)\right) \subset \bigcap_{k=1}^{\infty} G^{k m}(X) \\
& =\bigcap_{k=1}^{\infty}\left(G^{m}\right)^{k}(X)=\left\{x^{*}\right\} .
\end{aligned}
$$

Thus $\bigcap_{k=1}^{\infty} G^{k}(X)=\left\{x^{*}\right\}$. It follows that $G^{k}(x) \rightarrow x^{*}$ for all $x \in X$, this proves (b). By an elegant theorem of Janos [6], the conclusion of (c) follows. This completes the proof.

3. A few general remarks concerning Theorem 2 are in order. First, in Theorem 2 we have no information on rate of convergence with respect to the metric $d$. Second, unlike Theorem 1, the existence of fixed point in Theorem 2 cannot be guaranteed if we remove the continuity assumption, as the simple one-dimensional example $G(x):=1$, if $x=0 ; G(x):=x / 2$, if $x \in(0,1]$ shows. Third, if condition (2) is replaced by

$$
\begin{aligned}
d\left(G^{m}(x), G^{m}(y)\right)<\max \{ & d(x, y), d\left(x, G^{m}(x)\right), \\
& \left.d\left(y, G^{m}(y)\right), \frac{1}{2}\left(d\left(x, G^{m}(y)\right)+d\left(y, G^{m}(x)\right)\right)\right\}
\end{aligned}
$$

for all $x, y \in X$,

the existence $x^{*}$ in Theorem 2 can be seen as follows: The map $\psi(x):=d\left(x, G^{m}(x)\right)$ is continuous on the compact space $X$, hence $\psi$ takes its minimum value at some point $x^{*} \in X$. If $\psi\left(x^{*}\right)>0$, then $\psi\left(G\left(x^{*}\right)\right)<\psi\left(x^{*}\right)$ yielding a contradiction. Thus $G^{m}\left(x^{*}\right)=x^{*}$, and hence $G\left(x^{*}\right)=x^{*}$. (See Edelstein [4], and Hardy and Rogers [5].) Further, if condition (2) is replaced by

$$
\begin{array}{r}
d\left(G^{m}(x), G^{m}(y)\right)<\max \left\{d(x, y), \frac{1}{2}\left(d\left(x, G^{m}(x)\right)+d\left(y, G^{m}(y)\right)\right),\right. \\
\left.\frac{1}{2}\left(d\left(x, G^{m}(y)\right)+d\left(y, G^{m}(x)\right)\right)\right\},
\end{array}
$$

we can prove the existence $x^{*}$ in Theorem 2 without the continuity hypothesis on $G$, see [1]. Finally, one wonders whether a map $G$ on a compact space $(X, d)$ satisfying (2) may satisfy (1). The following simple example shows that this is, in general, not true.

EXAMPLE. Let $X:=[0,1]$ with the usual distance $|\cdot|$. Define

$$
G(x):=\frac{x}{1+x} \text { for } x \in[0,1] \text {. }
$$

Then $G$ satisfies condition (2) with $m=1$. However, $G$ does not satisfy (1); for otherwise there is an $\alpha \in(0,1)$ such that for all $x \in X$,

$$
\frac{x}{1+x}=|G(0)-G(x)| \leq \alpha \max \left\{x, 0, x-\frac{x}{1+x}, \frac{x}{1+x}\right\}=\alpha x .
$$

Hence $(1+x)^{-1} \leq \alpha$ for any $x \in X$ with $x \neq 0$. This is clearly impossible. 
4. AdDED in Proof. Let $(X, d)$ be a metric space and $D$ a nonempty bounded subset of $X$. Following Kuratowski, we define $\alpha(D)$, the measure of noncompactness of $D$, to be

$$
\inf \{l>0: D \text { can be covered by a finite number of sets of diameter } \leq l\} .
$$

If $\Omega$ is a nonempty subset of $X$ and $T$ a map of $\Omega$ into $X$, then $T$ is called condensing if $T$ is continuous on $\Omega$ and given any nonempty bounded set $S$ in $\Omega$, then $\alpha(T(S)<$ $\alpha(S)$ for $\alpha(S)>0$.

By the theorem of Janos, Ko and Tan [Theorem, Proc. Amer. Math. Soc. 76 (1979), 339-344], we can prove the following result, generalizing a result of Janos (see the corollary of Janos, Ko and Tan).

THEOREM 3. Let $(X, d)$ be a bounded complete metric space, and let $G: X \rightarrow X$. Suppose that there exists a positive integer $m$ such that $G^{m}$ is condensing and

$$
\begin{aligned}
d\left(G^{m}(x), G^{m}(y)\right) & \\
& <\max \left\{d(x, y), d\left(x, G^{m}(x)\right), d\left(y, G^{m}(y)\right), d\left(x, G^{m}(y)\right), d\left(y, G^{m}(x)\right)\right\}
\end{aligned}
$$

for all $x, y \in X$ with $x \neq y$. Then there exists a bounded complete metric $d^{*}$ on $X$ which is equivalent to $d$ such that $G$ is contractive with respect to $d^{*}$, i.e., $d^{*}(G(x), G(y))<d^{*}(x, y)$ for all $x, y \in X$ with $x \neq y$.

\section{REFERENCES}

1. Ming-Po Chen and Mau-Hsiang Shih, Fixed point theorems for point-to-point and point-to-set maps, J. Math. Anal. Appl. 71 (1979), 516-524.

2. S. C. Chu and J. B. Diaz, Remarks on a generalization of Banach's principle of contractive mappings, J. Math. Anal. Appl. 11 (1965), 440-446.

3. Lj. B. Ćirić, A generalization of Banach's contraction principle, Proc. Amer. Math. Soc. 45 (1974), 267-273.

4. M. Edelstein, On fixed points and periodic points under contractive mappings, J. London Math. Soc. 37 (1962), 74-79.

5. G. Hardy and T. Rogers, A generalization of a fixed point theorem of Reich, Canad. Math. Bull. 16 (1973), 201-206.

6. L. Janos, A converse of Banach's contraction theorem, Proc. Amer. Math. Soc. 16 (1967), 287289.

7. A. N. Kolmogorov and S. V. Fomin, Elements of the theory of functions and functional analysis, Metric and Normed Spaces, Vol. 1, transl. by L. Baron, Graylock Press, Rochester, N.Y., 1957.

8. P. R. Meyer, A converse to Banach's contraction theorem, J. Res. Nat. Bur. Standards 71B (1967), 73-76.

Department of Mathematics, Chung-Yuan University, Chung-Li, TaiWAN

Department of Mathematics, Central University, Chung-Li, TaiwaN 